\title{
Three-dimensional black-blood multi-contrast carotid imaging using compressed sensing: a repeatability study
}

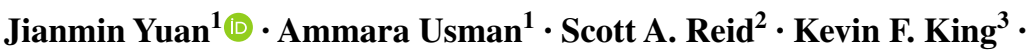 \\ Andrew J. Patterson ${ }^{4} \cdot$ Jonathan H. Gillard ${ }^{1}$ Martin J. Graves ${ }^{1,4}$
}

Received: 31 January 2017 / Revised: 9 June 2017 / Accepted: 16 June 2017 / Published online: 26 June 2017

(C) The Author(s) 2017. This article is an open access publication

\begin{abstract}
Objective The purpose of this work is to evaluate the repeatability of a compressed sensing (CS) accelerated multi-contrast carotid protocol at $3 \mathrm{~T}$.

Materials and methods Twelve volunteers and eight patients with carotid disease were scanned on a $3 \mathrm{~T}$ MRI scanner using a CS accelerated 3-D black-blood multi-contrast protocol which comprises $T_{1} \mathrm{w}, T_{2} \mathrm{w}$ and PDw without $\mathrm{CS}$, and with a CS factor of 1.5 and 2.0. The volunteers were scanned twice, the lumen/wall area and wall thickness were measured for each scan. Eight patients were scanned once, the inter/intra-observer reproducibility of the measurements was calculated.

Results In the repeated volunteer scans, the interclass correlation coefficient (ICC) for the wall area measurement using a CS factor of 1.5 in PDw, $T_{1} \mathrm{w}$ and $T_{2} \mathrm{w}$ were 0.95 , 0.81 , and 0.97 , respectively. The ICC for lumen area measurement using a CS factor of 1.5 in PDw, $T_{1} \mathrm{w}$ and $T_{2} \mathrm{w}$ were $0.96,0.92$, and 0.96 , respectively. In patients, the ICC for inter/intra-observer measurements of lumen/wall area, and wall thickness were all above 0.81 in all sequences. Conclusion The results show a CS accelerated 3-D blackblood multi-contrast protocol is a robust and reproducible
\end{abstract}

Jianmin Yuan

jy338@cam.ac.uk

1 Department of Radiology, School of Clinical Medicine, University of Cambridge, Level 5, Box 218, Addenbrooke's Hospital, Hills Rd, Cambridge CB2 0QQ, UK

2 GE Healthcare, Amersham, UK

3 GE Healthcare, Waukesha, WI, USA

4 Department of Radiology, Cambridge University Hospitals NHS Foundation Trust, Cambridge, UK method for carotid imaging. Future protocol design could use CS to reduce the scanning time.

Keywords Multi-contrast · Carotid MRI · Compressed sensing

\section{Introduction}

High-resolution magnetic resonance imaging (MRI) is a useful clinic method to assess carotid plaque vulnerability, due to its excellent soft tissue contrast $[1,2]$. A multicontrast MR protocol comprising PDw, $T_{1} \mathrm{w}$ and $T_{2} \mathrm{w}$ sequences can identify high-risk intraplaque components with high sensitivity and specificity [2]. Though still not used in clinical routine, the blood suppressed multi-contrast carotid MRI protocol has been used in previous research studies for plaque classification [2], component segmentation $[3,4]$ and also to determine the age of intraplaque hemorrhage [5].

Black-blood techniques have been used to suppress the blood signal in the lumen to improve vessel wall visualisation. This is usually achieved in 2-D imaging with the use of magnetisation preparation schemes such as double [6, 7], or quadruple inversion-recovery [8]. For 3-D imaging, non-selective preparation schemes such as motionsensitized driven-equilibrium (MSDE) $[9,10]$ or delay alternating with nutation for tailored excitation (DANTE) $[11,12]$ have been used. Volumetric (3-D) readout based on either fast-spin-echo [3, 13] or gradient-echo [12] allows the acquisition of near isotropic voxels, which could potentially improve the plaque component quantifications. However, one of the disadvantages of 3-D protocols in previous carotid studies is the long acquisition time (approximately 20-45 min), which could lead to poor patient compliance. 
Several techniques have been introduced to reduce the 3 -D acquisition time. Parallel imaging (PI) methods have been introduced, with the use of multi-channel coils [6, 14]. One of the studies has shown its ability to reduce the acquisition time by a factor of two whilst maintaining a high reproducibility for plaque quantitative measurement [6]. Dedicated pulse sequences have also been developed to acquire multiple contrast weightings in a single acquisition, such as the multi-contrast atherosclerosis characterization (MATCH) sequence, which can acquire hyper $T_{1} \mathrm{w}$, gray blood, and $T_{2} \mathrm{~W}$ images in a single 5-min sequence [15]. More recently, the method of compressed sensing (CS) has been introduced into MRI as an alternative method to accelerate image acquisition [16], and has been applied to carotid imaging [17-19]. These studies showed that the CS accelerated MERGE (motion-sensitizing driven equilibrium prepared 3-D rapid gradient echo) sequence could be used for rapid 3-D carotid wall imaging. Whilst the first two techniques (PI and MATCH) have been used for multicontrast purposes, the use of CS has not previously been validated in a multi-contrast protocol. The purpose of this work, therefore, is to evaluate the use of CS within a current standard carotid multi-contrast protocol. Volunteer scans were carried out to evaluate the interscan reproducibility and patients with carotid atherosclerotic diseases were recruited to validate inter/intra-observer reproducibility of the morphology measurements based on the CS accelerated sequences.

\section{Materials and methods}

\section{Study subjects}

This study had ethical approval and informed consent was obtained from each volunteer and patient. Twelve volunteers (eight men, mean age 34, range 24-55 years) and eight patients (four men, mean age 75 , range $72-87$ years) with a carotid artery stenosis greater than $50 \%$ on duplex ultrasound were scanned on a 3 T MRI system (MR 750, GE Healthcare, Waukesha, WI), using a four-channel phased-array neck coil (PACC, MachNet, Roden, The Netherlands). To evaluate the interscan reproducibility of the sequences, the 12 volunteers were scanned for a second time using the same protocol. The time interval between the two scans was 14 days (range 7-28 days).

\section{Compressed sensing}

The compressed sensing was achieved by using a Gaussian pseudo-random distribution undersampling pattern in $k$-space. The $32 \times 32$ area in the $k$-space center remained fully sampled to achieve high image quality. During the image reconstruction, the following objective function was applied:

$\hat{m}=\|\Psi m\|_{1}$ such that $\|F \hat{m}-y\|_{2}^{2} \leq \varepsilon$,

where $\psi$ is the sparsifying transform, which uses the gradient transform implemented as a nearest neighbor finite difference of the complex image $m, F$ is the fourier transform operator and $y$ is the acquired $k$-space data [16]. Fifteen iteration loops were used to minimize the penalty (L1-norm). In each of the iterations, the acquired $k$-space was subtracted back into the estimated $k$-space to maintain the data consistency. The CS sampling and reconstruction algorithm also enabled the combined use of parallel imaging, with the auto-calibrated reconstruction of the Cartesian data (ARC) [20] method, in order to reduce the overall examination time for both volunteers and patients. The investigation for the effects of varying CS acceleration factors alone, i.e., without the use of ARC, was evaluated using the $T_{1} \mathrm{w}$ acquisition. For the PDw and $T_{2} \mathrm{w}$ acquisitions, the CS was combined with an ARC acceleration of two in the phase encoding direction. The ARC and CS were combined sequentially with the CS algorithm applied as the first step and ARC as the second step. Lastly, a sumof-squares coil combination was performed to get the final image. Further details of the image acquisition and reconstruction can be found in the references [21-23].

\section{Imaging protocol}

All the subjects were imaged using a multi-contrast protocol listed in Table 1. Except for the patient scan, only a CS factor of 1.5 in PDw and $T_{2} \mathrm{w}$ was used due to limited scanning time. Coronal imaging slabs of the 3-D sequences were centered at the carotid bifurcation.

$T_{1} \mathrm{w}$ images were acquired by a DANTE [11] prepared 3-D FSE sequence. Images were acquired without CS acceleration, and with CS acceleration factor of 1.5 and 2.0. The scanning times were $3 \mathrm{~min} 16 \mathrm{~s}, 2 \mathrm{~min} 13 \mathrm{~s}$ and $1 \mathrm{~min}$ $41 \mathrm{~s}$, respectively. The parameters for DANTE preparation were: the number of pulses: $150 ; 3 \mathrm{G} / \mathrm{cm}$; gradient axes: $X, Y$ and $Z$; flip angle: $13^{\circ}$; DANTE pulse repetition time: $1 \mathrm{~ms}$. No parallel imaging was used for the $T_{1} \mathrm{w}$ images. $T_{2} \mathrm{~W}$ and PDw images were acquired using an iMSDE [9] prepared 3-D FSE sequence. The first-order moment $\left(m_{1}\right)$ was empirically set to $412 \mathrm{mTms}^{2} / \mathrm{m}$. The scanning time was $3 \min 36 \mathrm{~s}, 2 \min 36 \mathrm{~s}$ and $2 \min 7 \mathrm{~s}$ for both of the sequences without CS, and with CS factors of 1.5 and 2.0. The CS and ARC were combined in a sequential way [23]. Fat suppression was performed using an Adiabatic SPectral Inversion Recovery (ASPIR) pulse. The acquired resolution for $T_{1} \mathrm{w}, T_{2} \mathrm{w}$ and PDw was $0.6 \times 0.6 \times 1.4 \mathrm{~mm}^{3}$. Electrocardiography (ECG) gating was not used in the protocol. 
Table 1 Scanning parameters for the multi-contrast protocol

\begin{tabular}{|c|c|c|c|c|}
\hline Contrast & Time-of-flight & $T_{1} \mathrm{w}$ & PDw & $T_{2} \mathrm{~W}$ \\
\hline Sequence & 3-D SPGR & 3-D FSE & 3-D FSE & 3-D FSE \\
\hline Acquisition direction & Axial & Coronal & Coronal & Coronal \\
\hline Blood suppression & - & DANTE & iMSDE & iMSDE \\
\hline Echo time (ms) & 2.2 & 16.9 & 21.6 & 51.6 \\
\hline Repetition time (ms) & 5.9 & 540 & 2000 & 2000 \\
\hline Flip angle $\left(^{\circ}\right)$ & 20 & Variable flip angle & Variable flip angle & Variable flip angle \\
\hline $\mathrm{FOV}\left(\mathrm{mm}^{3}\right)$ & $140 \times 140 \times 64$ & $140 \times 140 \times 67$ & $140 \times 140 \times 56$ & $140 \times 140 \times 56$ \\
\hline Acquisition matrix & $256 \times 256 \times 32$ & $224 \times 224 \times 48$ & $224 \times 224 \times 40$ & $224 \times 224 \times 40$ \\
\hline ARC Parallel Imaging (phase $\times$ slice) & - & - & $2 \times 1$ & $2 \times 1$ \\
\hline CS acceleration & Non-CS & Non-CS/1.5/2.0 & Non-CS/1.5/2.0 & Non-CS/1.5/2.0 \\
\hline Acquisition time & $1: 35$ & $3: 16 / 2: 13 / 1: 41$ & $3: 36 / 2: 36 / 2: 07$ & $3: 36 / 2: 36 / 2: 07$ \\
\hline
\end{tabular}

\section{Image analysis}

The acquired multi-contrast images were first reformatted into the axial plane, and then interpolated into a voxel size of $0.2 \times 0.2 \times 0.3 \mathrm{~mm}^{3}$, using MATLAB (The MathWorks, Inc., Natick, MA). Carotid artery lumen and outer wall boundaries were manually drawn by an experienced observer who has more than 2 years' experience in carotid imaging, using a Food and Drug Administration (FDA) proved DICOM viewer (OsiriX 5.5.2, Pixmeo, Geneva, Switzerland).

For the volunteer images, five contiguous slices in the common, internal and external carotid artery (CCA, ICA and ECA), $5 \mathrm{~mm}$ below and $5 \mathrm{~mm}$ above the bifurcation were used for morphological measurements. The lumen area was defined as the area inside the lumen contour, and the wall area was defined as the area between the outer wall boundary and inner lumen contour. The mean radius of the lumen and outer wall was calculated by simplifying the geometry as a circle with the same area. The mean wall thickness was determined as the difference between lumen radius and outer wall radius. The wall-lumen sharpness was also calculated for each CS factor in the $T_{1} \mathrm{w}$ sequences [24]. To calculate the walllumen sharpness, a line profile perpendicular from the vessel wall to the lumen was analysed. Due to the blood suppression, the lowest signal pixel (in the blood) in the line profile is set to zero and the highest intensity pixel (in the wall) is set to be 1 . The distance between the pixel intensity at 0.2 and 0.8 was calculated. The image sharpness was defined as one over the distance. For the patient images, the vessel wall and lumen boundaries were manually drawn on each of the slices containing atherosclerotic plaque. The plaque was defined as a focal

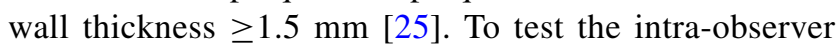
reproducibility, the $T_{1} \mathrm{w}, T_{2} \mathrm{w}$ and PDw images with a CS factor of 1.5 were analysed twice by the same observer.
To test the inter-observer reproducibility, the $T_{1} \mathrm{w}, T_{2} \mathrm{w}$ and PDw images were analysed by a second observer who also has more than 2 years of carotid imaging, and the results were compared with the first observer. Both of the observers made their measurements independently for each time, and were blinded to the subjects' clinical information.

\section{Statistical analysis}

Interclass correlations (ICCs) and coefficient of variation $(\mathrm{CoV})$ were calculated to evaluate the agreement of wall/ lumen area and wall thickness measurements between two volunteer scans. The $\mathrm{CoV}$ is defined as the ratio of the standard deviation of the difference between the two measurements to the mean value

$\operatorname{CoV}=\frac{\operatorname{sd}\left(x_{1 i}-x_{2 i}\right)}{\left(\sum_{i=1}^{n} \frac{x_{1 i}+x_{2 i}}{2}\right) / n}$

where $x_{1}$ and $x_{2}$ are the first and second measurement, $n$ is the number of subjects, $i=1,2, \ldots n$ and sd is the standard deviation.

The measurement differences from $T_{1} \mathrm{w}$ images between two scans were analysed using the Bland-Altman method. The inter/intra-observer variabilities from patients' scans were also evaluated using the ICC and CoV. An ICC value above 0.75 was considered as excellent agreement. The $0.40-075$ was good agreement and below 0.40 was poor agreement. A two-tailed paired student's $t$ test was used to compare the wall thickness measurements between non-CS and CS accelerated $T_{1} \mathrm{w}$ sequences in patients. Statistical significance was defined if $p<0.05$. Continuous data were presented as mean $\pm \mathrm{sd}$. The statistical analysis was performed using $\mathrm{R}$ (version 3.2.2). 
Table 2 ICC $(95 \%$ CI $)$ and $\mathrm{CoV}$ of the scan-rescan measurements of the multicontrast sequences using no CS, CS factor of 1.5 and 2.0 from volunteers

\begin{tabular}{llll}
\hline & No CS & CS 1.5 & CS 2.0 \\
\hline ICC & & & \\
$T_{1} \mathrm{w}$ & & & \\
Wall area & $0.94(0.90-0.97)$ & $0.81(0.69-0.88)$ & $0.81(0.70-0.89)$ \\
Lumen area & $0.95(0.91-0.97)$ & $0.92(0.87-0.95)$ & $0.91(0.85-0.95)$ \\
$T_{2} \mathrm{w}$ & & $0.81(0.74-0.86)$ \\
Wall area & $0.96(0.94-0.97)$ & $0.97(0.94-0.98)$ & $0.91(0.81-0.96)$ \\
Lumen area & $0.97(0.93-0.98)$ & $0.96(0.94-0.98)$ & \\
PDw & & & $0.82(0.80-0.92)$ \\
Wall area & $0.91(0.79-0.97)$ & $0.95(0.92-0.97)$ & $0.91(0.81-0.95)$ \\
Lumen area & $0.95(0.90-0.96)$ & $0.96(0.92-0.97)$ & \\
CoV & & & $24 \%$ \\
$T_{1} \mathrm{w}$ & & $26 \%$ & $17 \%$ \\
Wall area & $12 \%$ & $16 \%$ & $20 \%$ \\
Lumen area & $11 \%$ & & $17 \%$ \\
$T_{2} \mathrm{w}$ & & $14 \%$ & $22 \%$ \\
Wall area & $13 \%$ & $15 \%$ & $18 \%$ \\
Lumen area & $14 \%$ & $14 \%$ & \\
PDw & & & \\
Wall area & $19 \%$ & $12 \%$ & \\
Lumen area & & & \\
\hline
\end{tabular}

\section{Results}

All of the twelve volunteers completed the scans. Seven out of eight patients completed the scan. One patient did not finish the scan due to discomfort.
Figure 1 shows the Bland-Altman plots of lumen and wall area measurement of volunteer $T_{1} \mathrm{w}$ images between two scans with non-CS, CS factor of 1.5 and 2.0. The ICC $(95 \% \mathrm{CI})$ and $\mathrm{CoV}$ for lumen and wall area measured from the repeated volunteer scans using the three
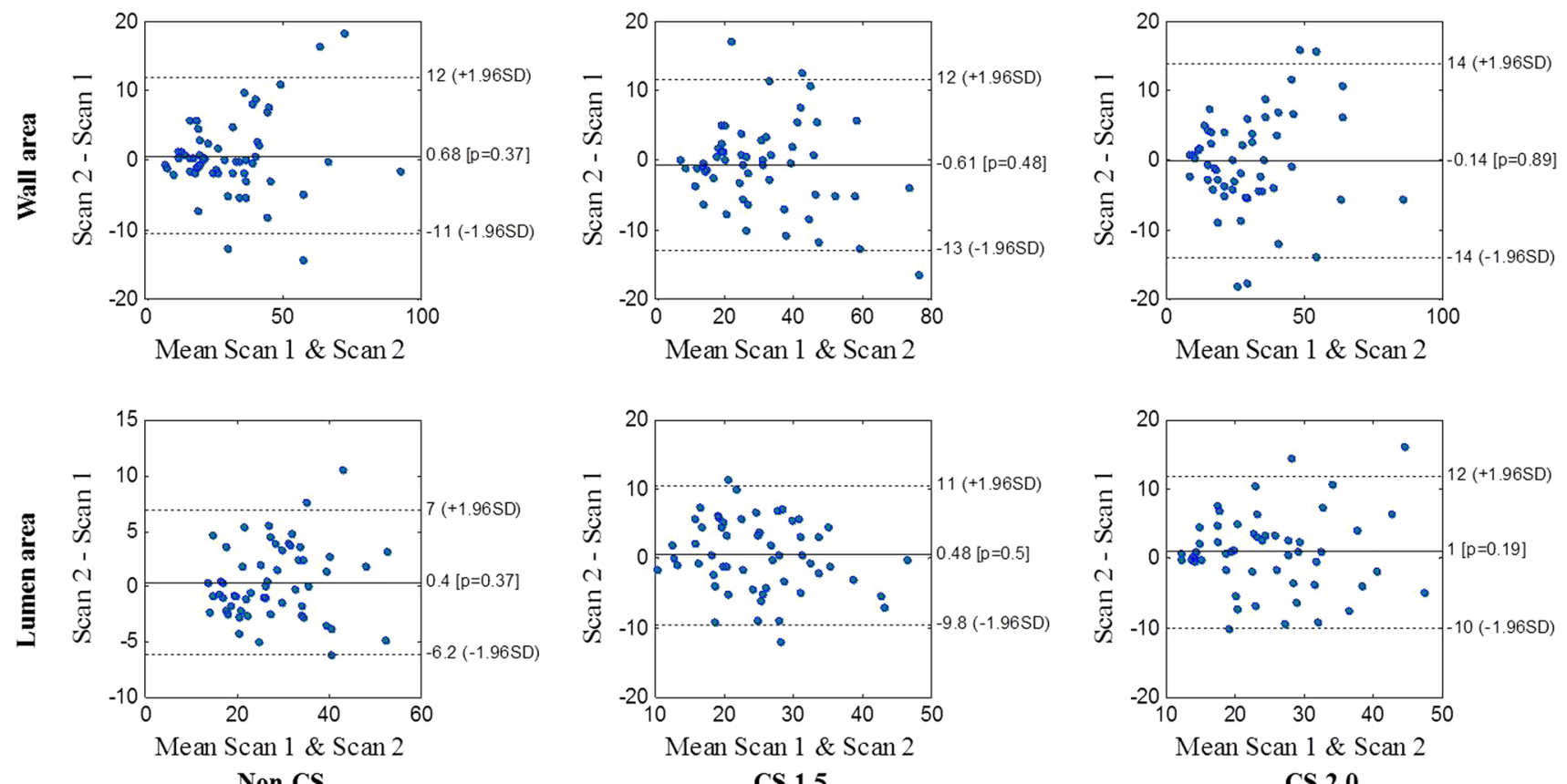

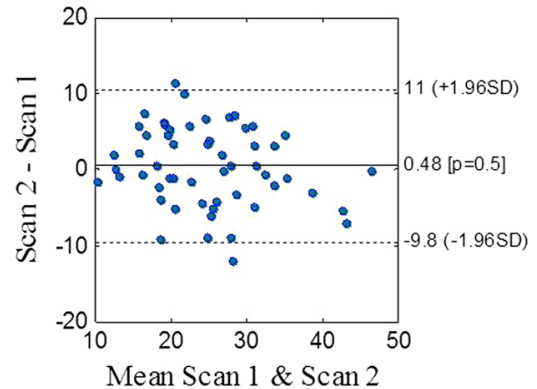

CS 1.5

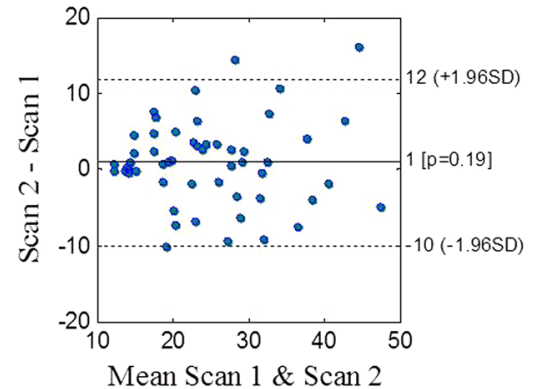

CS 2.0

Fig. 1 Bland-Altman plots of volunteer lumen and wall area measurements from $T_{1}$ w sequences between two repeated scans (all units mm ${ }^{2}$ ) 


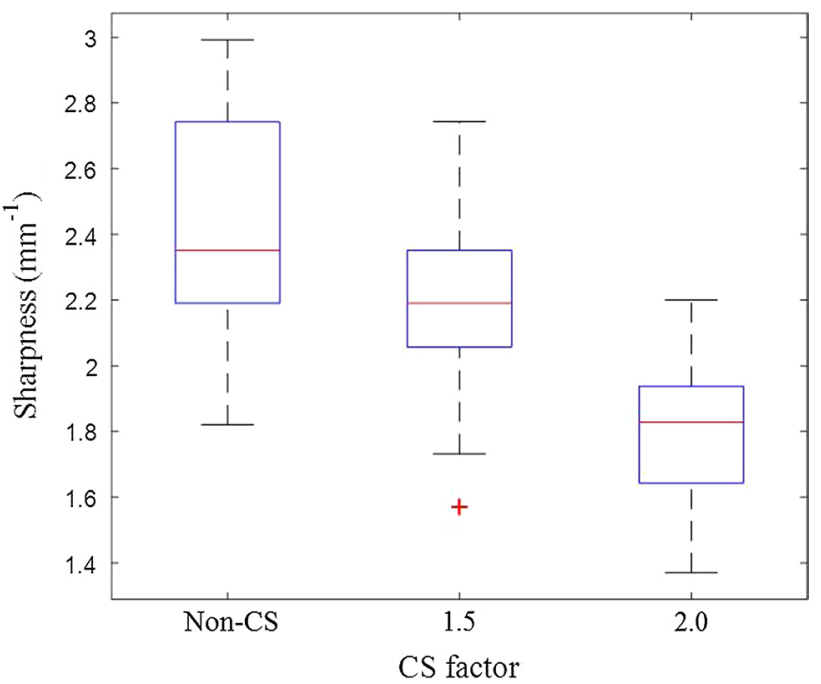

Fig. 2 The wall-lumen sharpness measured from volunteer $T_{1} \mathrm{w}$ images with different CS factors

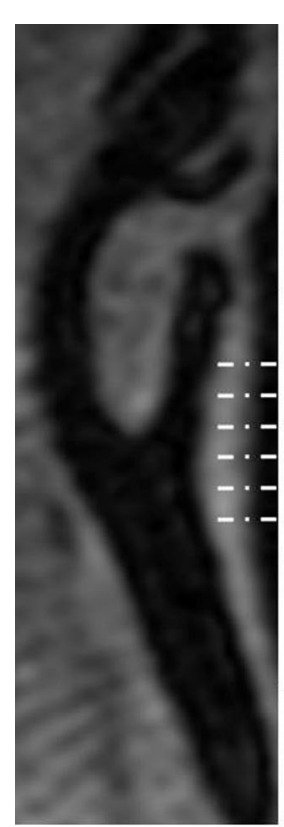

Contrast

CS factor
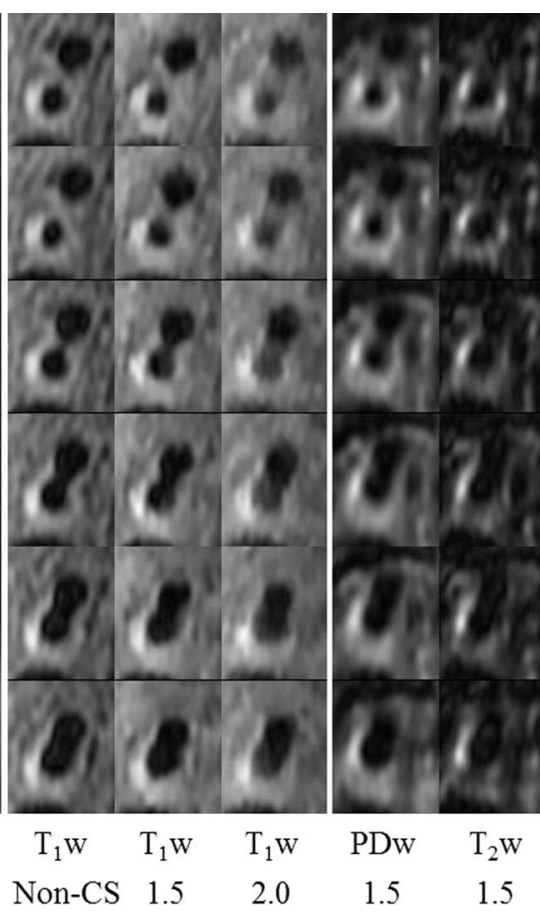

Fig. 3 Multi-contrast carotid images of a 80-year-old male. The plaque contains an intraplaque haemorrhage

multi-contrast sequences with different CS factors are shown in Table 2. Excellent correlation of wall/lumen area measurement were found between two scans (all ICCs $>0.80$ ). Figure 2 shows the wall-lumen sharpness calculated from the $T_{1} \mathrm{w}$ volunteer images. The sharpness decreases with increasing CS factor.

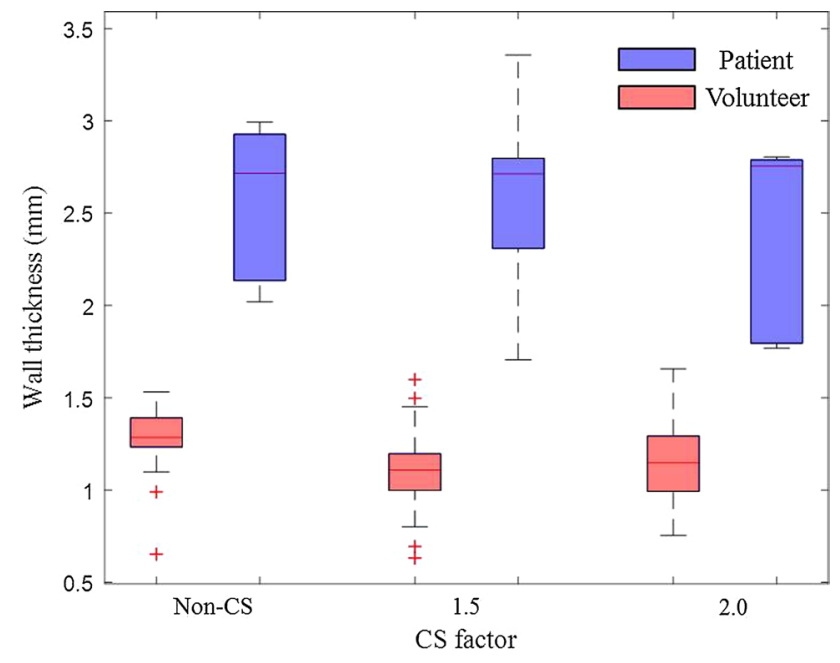

Fig. 4 Volunteer and patient's wall thickness at different CS factors of $T_{1} \mathrm{w}$ images

Figure 3 shows an example of a patient image with intraplaque hemorrhage. From the $T_{1} \mathrm{w}$ images, the wall-lumen and outer wall boundaries become blurred with increasing CS factor. However, for the wall thickness measurement, there are no significant differences comparing the CS accelerated sequence with the non-CS sequence (Non-CS vs. CS1.5: $2.43 \pm 0.57 \mathrm{~mm}$ vs. $2.58 \pm 0.87 \mathrm{~mm}, p=0.58$; Non-CS vs. CS2.0: $2.43 \pm 0.57 \mathrm{~mm}$ vs. $2.39 \pm 0.85 \mathrm{~mm}$, $p=0.59)$. When compared to the volunteers, the patients have a thicker wall thickness $(p<0.05)$, as shown in Fig. 4 .

Table 3 shows the ICC with 95\% CI and CoV of the intra/inter-observer reproducibility in measuring the lumen/wall area and wall thickness in multi-contrast sequences with a CS factor of 1.5 from the patients. All the ICCs were above 0.81 . Table 4 shows the intraobserver reproducibility of three different CS factors using $T_{1} \mathrm{w}$ sequence in patient scans. The ICCs were above 0.83 .

\section{Discussion}

This study demonstrates, for the first time, the usefulness of CS acceleration in a multi-contrast black-blood carotid protocol to reduce the overall acquisition time at $3 \mathrm{~T}$. The results show that CS accelerated sequences have a good scan-rescan reproducibility in carotid morphological measurement in volunteers, and good inter/intraobserver reproducibility of morphological measurement in patients.

Multi-contrast MRI has been used widely in assessing plaque components and vulnerability $[2,3,12,26-$ 34]. Originally this was performed at $1.5 \mathrm{~T}$ [2, 26-30], 
Table 3 ICC (95\% CI) and $\mathrm{CoV}$ for the intra/inter-observer measurements of the multicontrast protocol using CS factor of 1.5 from patients' scans

\begin{tabular}{llll}
\hline & PDw & $T_{1} \mathrm{w}$ & $T_{2} \mathrm{~W}$ \\
\hline $\begin{array}{l}\text { ICC } \\
\text { Intra-observer }\end{array}$ & & \\
$\quad$ Wall area & $0.86(0.51-0.98)$ & $0.84(0.50-0.96)$ & $0.82(0.43-0.97)$ \\
Lumen area & $0.98(0.95-0.99)$ & $0.98(0.92-1.00)$ & $0.98(0.96-0.99)$ \\
Wall thickness & $0.98(0.96-0.99)$ & $0.86(0.57-0.96)$ & $0.97(0.95-0.99)$ \\
Inter-observer & & & \\
Wall area & $0.84(0.50-0.96)$ & $0.87(0.40-0.98)$ & $0.86(0.51-0.97)$ \\
Lumen area & $0.98(0.95-0.99)$ & $0.95(0.77-0.99)$ & $0.98(0.93-0.99)$ \\
Wall thickness & $0.98(0.94-0.99)$ & $0.92(0.63-0.97)$ & $0.95(0.89-0.99)$ \\
CoV & & & \\
Intra-observer & & & $27 \%$ \\
Wall area & $22 \%$ & $25 \%$ & $12 \%$ \\
Lumen area & $13 \%$ & $14 \%$ & $14 \%$ \\
$\quad$ Wall thickness & $12 \%$ & $19 \%$ & \\
Inter-observer & & & $20 \%$ \\
Wall area & $25 \%$ & $17 \%$ & $14 \%$ \\
Lumen area & $13 \%$ & $15 \%$ & $16 \%$ \\
Wall thickness & $14 \%$ & & \\
\hline
\end{tabular}

Table 4 ICC (95\% CI) and CoV for the intra-observer measurements of the $T_{1} \mathrm{~W}$ sequences using no CS, CS factor of 1.5 and 2.0 from patients' scans

\begin{tabular}{llll}
\hline & No CS & CS 1.5 & CS 2.0 \\
\hline ICC & & & \\
Wall area & $0.95(0.79-0.99)$ & $0.84(0.50-0.96)$ & $0.89(0.54-0.98)$ \\
Lumen area & $0.99(0.95-1.00)$ & $0.98(0.92-1.00)$ & $0.93(0.66-0.99)$ \\
Wall thickness & $0.90(0.60-0.98)$ & $0.86(0.57-0.96)$ & $0.93(0.67-0.99)$ \\
CoV & & & \\
Wall area & $15 \%$ & $25 \%$ & $19 \%$ \\
Lumen area & $11 \%$ & $14 \%$ & $18 \%$ \\
Wall thickness & $14 \%$ & $19 \%$ & $13 \%$ \\
\hline
\end{tabular}

however the studies are now more commonly performed at $3 \mathrm{~T}$ due to the superior signal-to-noise ratio (SNR) and contrast-to-noise ratio (CNR) [31-33, 35-37]. In addition, the development of 3-D sequences allows for larger coverage, better through-plane resolution, higher scanning efficiency, less motion artefact and more precise plaque segmentation $[3,12,15]$. Whilst acceleration techniques such as PI have been introduced and validated [6], the use of CS has not previously been validated for the multi-contrast protocol. The CS technique has previously been used in carotid studies to reduce the acquisition time in sequences such as MERGE [17-19, 38] and black-blood dynamic contrast enhanced MRI [39]. The studies showed that CS can be used to (1) reduce the acquisition time without significantly impacting on the diagnostic quality, (2) reduce motion artefacts and
(3) achieve higher temporal resolution in dynamic imaging. Previous studies have also demonstrated that CS produces better image quality than PI [40], and that the combination of CS and PI can achieve even better image quality than either of the techniques used alone [41]. This study has, for the first time, reported the scan-rescan reproducibility and intra-inter observer repeatability of carotid morphological measurement in a multi-contrast protocol. The results showed that wall area, lumen area and wall thickness is reproducible in a CS accelerated multi-contrast protocol, using a productised reconstruction with accepted reconstruction time.

The results from this study show that the wall-lumen sharpness decreases (Fig. 2), and the CoV of volunteer scan-rescan measurements increases with increasing CS factor (Table 2). This indicates that the use of CS brings additional uncertainty in the morphological measurement. Table 2 indicates that with either no or small CS factors (CS 1.5), the $\mathrm{CoV}$ of morphological measurements in repeatability scans is acceptably low.

There are several limitations in this study. Firstly, the number of patients is quite small. Only eight patients were scanned using the current multi-contrast protocol. Therefore, only limited plaque components and examples were investigated. However, good reproducibility of the quantitative analysis from the volunteer scan demonstrates the feasibility of applying the CS-accelerated protocol for a future large-scale patient study. The second limitation is that a coronal acquisition with anisotropic resolution was used in this study as a trade-off between blood suppression, image SNR, scanning time and coverage. This may 
lead to limitations in the wall thickness measurements and potentially affect the plaque characterization in the reconstructed axial image. Nevertheless, the result from the current scanning settings showed good repeatability of the wall thickness measurements. Future optimisation should consider the use of isotropic resolution acquisitions for better plaque characterization. The third limitation is that the CS reconstruction method used in this study was a vendor provided on-line algorithm, which was optimised for clinically acceptable reconstruction times. Further optimisation of the acquisition and reconstruction parameters, or to even use the multi-contrast sequences for joint reconstruction [42], could potentially improve the image quality, albeit probably at the expense of increased reconstruction time. Fourthly, considering the limited scanning time in practice, ARC acceleration of $2 \times 1$ and only a single CS factor of 1.5 was used for PDw and $T_{2} \mathrm{w}$ sequences. The results from the $T_{1}$ w may not necessarily be extrapolated to these two contrast weighted images. The last limitation is that a fixed echo train length was used in this study, as we primarily considered the CS as an additional feature to the existing optimised protocol setup. Longer echo train length with optimised flip angle schemes could further reduce the acquisition time, but this is outside the scope of this study.

\section{Conclusion}

In conclusion, this study shows that the current 3-D carotid black-blood multi-contrast protocol could be accelerated by CS, in addition to PI, with robust and reproducible morphology measurements. With the current protocol setup, a CS factor of 1.5 in combination with a parallel imaging acceleration factor of two can be used for a multi-contrast protocol.

\begin{abstract}
Authors' contribution JY participated in the study design, contributed to data acquisition, analysis, interpretation, and drafted the manuscript. AU was involved in study design, recruiting the patients and revising the manuscript. AJP, JHG and MJG designed the study, supervised the data acquisition, analysis, interpretation and prepared the manuscript. SAR and KFK were involved in sequence development, image processing and revising the manuscript.
\end{abstract}

\section{Compliance with ethical standards}

Funding The project was supported by the Addenbrooke's Charitable Trust and the NIHR Comprehensive Biomedical Research Centre award to Cambridge University Hospitals NHS Foundation Trust in partnership with the University of Cambridge.

Conflict of interest Scott Reid and Kevin King are employees of GE Healthcare.
Ethical approval All procedures performed in studies involving human participants were in accordance with the ethical standards of the institutional and/or national research committee and with the 1964 Helsinki Declaration and its later amendments or comparable ethical standards.

Informed consent Informed consent was obtained from all individual participants included in the study.

Open Access This article is distributed under the terms of the Creative Commons Attribution 4.0 International License (http://crea tivecommons.org/licenses/by/4.0/), which permits unrestricted use, distribution, and reproduction in any medium, provided you give appropriate credit to the original author(s) and the source, provide a link to the Creative Commons license, and indicate if changes were made.

\section{References}

1. Saam T, Hatsukami TS, Takaya N, Chu B, Underhill H, Kerwin WS, Cai J, Ferguson MS, Yuan C (2007) The vulnerable, or high-risk, atherosclerotic plaque: noninvasive $\mathrm{mr}$ imaging for characterization and assessment 1. Radiology 244(1):64-77

2. Cai J-M, Hatsukami TS, Ferguson MS, Small R, Polissar NL, Yuan C (2002) Classification of human carotid atherosclerotic lesions with in vivo multicontrast magnetic resonance imaging. Circulation 106(11):1368-1373

3. Liu W, Balu N, Sun J, Zhao X, Chen H, Yuan C, Zhao H, Xu J, Wang G, Kerwin WS (2012) Segmentation of carotid plaque using multicontrast 3D gradient echo MRI. J Magn Reson Imaging 35(4):812-819

4. Yoneyama T, Sun J, Hippe DS, Balu N, Xu D, Kerwin WS, Hatsukami TS, Yuan C (2016) In vivo semi-automatic segmentation of multicontrast cardiovascular magnetic resonance for prospective cohort studies on plaque tissue composition: initial experience. Int J Cardiovasc Imaging 32(1):73-81

5. Chu B, Kampschulte A, Ferguson MS, Kerwin WS, Yarnykh VL, O'Brien KD, Polissar NL, Hatsukami TS, Yuan C (2004) Hemorrhage in the atherosclerotic carotid plaque: a high-resolution MRI study. Stroke 35(5):1079-1084

6. Saam T, Raya JG, Cyran CC, Bochmann K, Meimarakis G, Dietrich O, Clevert DA, Frey U, Yuan C, Hatsukami TS (2009) High resolution carotid black-blood 3T MR with parallel imaging and dedicated 4-channel surface coils. J Cardiovasc Magn Reson 11(1): 1

7. Edelman RR, Chien D, Kim D (1991) Fast selective black blood MR imaging. Radiology 181(3):655-660

8. Yarnykh VL, Yuan C (2002) T1-insensitive flow suppression using quadruple inversion-recovery. Magn Reson Med 48(5):899-905

9. Wang J, Yarnykh VL, Hatsukami T, Chu B, Balu N, Yuan C (2007) Improved suppression of plaque-mimicking artifacts in black-blood carotid atherosclerosis imaging using a multislice motion-sensitized driven-equilibrium (MSDE) turbo spin-echo (TSE) sequence. Magn Reson Med 58(5):973-981

10. Wang J, Yarnykh VL, Yuan C (2010) Enhanced image quality in black-blood MRI using the improved motion-sensitized driven-equilibrium (iMSDE) sequence. J Magn Reson Imaging 31(5):1256-1263

11. Li L, Miller KL, Jezzard P (2012) DANTE-prepared pulse trains: a novel approach to motion-sensitized and 
motion-suppressed quantitative magnetic resonance imaging. Magn Reson Med 68(5):1423-1438

12. Li L, Chai JT, Biasiolli L, Robson MD, Choudhury RP, Handa AI, Near J, Jezzard P (2014) Black-blood multicontrast imaging of carotid arteries with DANTE-prepared 2D and 3D MR imaging. Radiology 273(2):560-569

13. Zhu C, Graves MJ, Yuan J, Sadat U, Gillard JH, Patterson AJ (2014) Optimization of improved motion-sensitized drivenequilibrium (iMSDE) blood suppression for carotid artery wall imaging. J Cardiovasc Magn Reson 16:61

14. Sumi T, Sumi M, Van Cauteren M, Kimura Y, Nakamura T (2007) Parallel imaging technique for the external carotid artery and its branches: comparison of balanced turbo field echo, phase contrast, and time-of-flight sequences. J Magn Reson Imaging 25(5):1028-1034

15. Fan Z, Yu W, Xie Y, Dong L, Yang L, Wang Z, Conte AH, Bi X, An J, Zhang T (2014) Multi-contrast atherosclerosis characterization (MATCH) of carotid plaque with a single 5-min scan: technical development and clinical feasibility. J Cardiovasc Magn Reson 16:53

16. Lustig M, Donoho D, Pauly JM (2007) Sparse MRI: the application of compressed sensing for rapid MR imaging. Magn Reson Med 58(6):1182-1195

17. Li B, Li H, Li J, Zhang Y, Wang X, Zhang J, Dong L, Fang J (2015) Relaxation enhanced compressed sensing three-dimensional black-blood vessel wall MR imaging: preliminary studies. Magn Reson Imaging 33(7):932-938

18. Li B, Dong L, Chen B, Ji S, Cai W, Wang Y, Zhang J, Zhang Z, Wang X, Fang J (2013) Turbo fast three-dimensional carotid artery black-blood MRI by combining three-dimensional MERGE sequence with compressed sensing. Magn Reson Med 70(5):1347-1352

19. Makhijani MK, Balu N, Yamada K, Yuan C, Nayak KS (2012) Accelerated 3D MERGE carotid imaging using compressed sensing with a hidden Markov tree model. J Magn Reson Imaging 36(5):1194-1202

20. Beatty P, Brau A, Chang S, Joshi S, Michelich C, Bayram E, Nelson T, Herfkens R, Brittain J (2007) A method for autocalibrating 2-D accelerated volumetric parallel imaging with clinically practical reconstruction times. In: Proceedings of the 15th scientific meeting, International Society for Magnetic Resonance in Medicine, Berlin, p 1749

21. King K, Xu D, Brau A, Lai P, Beatty P, Marinelli L (2010) A new combination of compressed sensing and data driven parallel imaging. In: Proceedings of the 18th scientific meeting, International Society for Magnetic Resonance in Medicine, Stockholm, p 4881

22. Yuan J, Usman A, Reid SA, King KF, Patterson AJ, Gillard JH, Graves MJ (2017) Three-dimensional black-blood T2 mapping with compressed sensing and data-driven parallel imaging in the carotid artery. Magn Reson Imaging 37:62-69

23. Pandit P, Rivoire J, King K, Li X (2016) Accelerated T1 $\rho$ acquisition for knee cartilage quantification using compressed sensing and data-driven parallel imaging: a feasibility study. Magn Reson Med 75(3):1256-1261

24. Larson AC, Kellman P, Arai A, Hirsch GA, McVeigh E, Li D, Simonetti OP (2005) Preliminary investigation of respiratory self-gating for free-breathing segmented cine MRI. Magn Reson Med 53(1):159-168

25. Kitamura A, Iso H, Imano H, Ohira T, Okada T, Sato S, Kiyama M, Tanigawa T, Yamagishi K, Shimamoto T (2004) Carotid intima-media thickness and plaque characteristics as a risk factor for stroke in Japanese elderly men. Stroke 35(12):2788-2794

26. Kang X, Polissar NL, Han C, Lin E, Yuan C (2000) Analysis of the measurement precision of arterial lumen and wall areas using high-resolution MRI. Magn Reson Med 44(6):968-972
27. Yuan C, Mitsumori LM, Ferguson MS, Polissar NL, Echelard D, Ortiz G, Small R, Davies JW, Kerwin WS, Hatsukami TS (2001) In vivo accuracy of multispectral magnetic resonance imaging for identifying lipid-rich necrotic cores and intraplaque hemorrhage in advanced human carotid plaques. Circulation 104(17):2051-2056

28. Saam T, Kerwin WS, Chu B, Cai J, Kampschulte A, Hatsukami TS, Zhao X-Q, Polissar NL, Neradilek B, Yarnykh VL (2005) Sample size calculation for clinical trials using magnetic resonance imaging for the quantitative assessment of carotid atherosclerosis. J Cardiovasc Magn Reson 7(5):799-808

29. Cappendijk VC, Cleutjens KB, Kessels AG, Heeneman S, Schurink GWH, Welten RJ, Mess WH, Daemen MJ, van Engelshoven JM, Kooi ME (2005) Assessment of human atherosclerotic carotid plaque components with multisequence MR imaging: initial experience 1. Radiology 234(2):487-492

30. Chu B, Zhao XQ, Saam T, Yarnykh VL, Kerwin WS, Flemming KD, Huston J, Insull W, Morrisett JD, Rand SD (2005) Feasibility of in vivo, multicontrast-weighted MR imaging of carotid atherosclerosis for multicenter studies. J Magn Reson Imaging 21(6):809-817

31. Yarnykh VL, Terashima M, Hayes CE, Shimakawa A, Takaya N, Nguyen PK, Brittain JH, McConnell MV, Yuan C (2006) Multicontrast black-blood MRI of carotid arteries: comparison between 1.5 and 3 tesla magnetic field strengths. J Magn Reson Imaging 23(5):691-698

32. Li F, Yarnykh VL, Hatsukami TS, Chu B, Balu N, Wang J, Underhill HR, Zhao X, Smith R, Yuan C (2010) Scan-rescan reproducibility of carotid atherosclerotic plaque morphology and tissue composition measurements using multicontrast MRI at 3T. J Magn Reson Imaging 31(1): 168-176

33. Young V, Patterson A, Tunnicliffe E, Sadat U, Graves M, Tang T, Priest A, Kirkpatrick P, Gillard J (2014) Signal-to-noise ratio increase in carotid atheroma MRI: a comparison of 1.5 and $3 \mathrm{~T}$. Br J Radiol 85(1015):937-944

34. Yuan J, Usman A, Das T, Patterson A, Gillard J, Graves M (2016) Imaging carotid atherosclerosis plaque ulceration: comparison of advanced imaging modalities and recent developments. Am J Neuroradiol. doi:10.3174/ajnr.A5026

35. Kröner E, Westenberg J, van der Geest R, Brouwer N, Doornbos J, Kooi M, van der Wall E, Lamb H, Siebelink H (2013) High field carotid vessel wall imaging: a study on reproducibility. Eur J Radiol 82(4):680-685

36. Roberts JA, Kim S-E, Yoon H-C, McNally JS, Hadley JR, Findeiss LK, Treiman GS, Parker DL (2012) reproducibility of lumen and vessel wall measurements in carotid magnetic resonance imaging. Open Cardiovasc Thorac Surg J 5:1-7

37. Zhou Z, Li R, Zhao X, He L, Wang X, Wang J, Balu N, Yuan C (2015) Evaluation of 3D multi-contrast joint intra-and extracranial vessel wall cardiovascular magnetic resonance. J Cardiovasc Magn Reson 17(1):41

38. Li B, Li H, Kong H, Dong L, Zhang J, Fang J (2017) Compressed sensing based simultaneous black-and gray-blood carotid vessel wall MR imaging. Magn Reson Imaging 38:214-223

39. Zhou Z-W, Sharif B, Fan Z, Xie Y, Li D (2014) High spatial and temporal resolution black-blood dynamic contrast-enhanced carotid artery wall MRI using compressed sensing. J Cardiovasc Magn Reson 16(S1):W40

40. Akçakaya M, Basha TA, Chan RH, Manning WJ, Nezafat R (2014) Accelerated isotropic sub-millimeter whole-heart coronary MRI: compressed sensing versus parallel imaging. Magn Reson Med 71(2):815-822

41. King K (2008) Combining compressed sensing and parallel imaging. In: Proceedings of the 16th annual meeting, International Society for Magnetic Resonance in Medicine, Toronto, p 1488

42. Bilgic B, Goyal VK, Adalsteinsson E (2011) Multi-contrast reconstruction with Bayesian compressed sensing. Magn Reson Med 66(6):1601-1615 